\title{
Tratamento não operatório de fraturas desviadas da extremidade proximal do úmero em idosos: Correlação entre os desvios e os resultados clínicos*
}

\section{Non-operative treatment for displaced proximal humeral fractures in elderly patients: Correlation between deviations and clinical outcomes}

\author{
Mauro Emilio Conforto Gracitelli1 ${ }^{10}$ \\ Gustavo Jum Yamamoto ${ }^{1}$ Eduardo Angeli Malavolta1잉 \\ Fernando Brandão Andrade-Silva1 ${ }^{10}$ \\ Kodi Edson Kojima1이 Arnaldo Amado Ferreira Neto ${ }^{1}{ }^{10}$
}

${ }^{1}$ Grupo de Ombro e Cotovelo, Instituto de Ortopedia e Traumatologia, Hospital das Clinicas HCFMUSP, Faculdade de

Medicina, Universidade de São Paulo, São Paulo, SP, Brasil

Endereço para correspondência Mauro E. C. Gracitelli, Ph.D, Rua Dr. Ovídio Pires de Campos, $333,3^{\circ}$ andar, Cerqueira Cesar, São Paulo, SP, 05403-010, Brasil (e-mail: mgracitelli@gmail.com).

Rev Bras Ortop 2022;57(2):273-281

\section{Resumo \\ Objetivos Descrever o resultado funcional do tratamento não operatório de fraturas desviadas da extremidade proximal do úmero (FEPU) pela escala da American Shoulder and Elbow Surgeons (ASES, na sigla em inglês) após 12 meses e avaliar se as diferentes classificações e medidas radiográficas iniciais têm correlação com os resultados clínicos. \\ Métodos Foram avaliados em tempos padronizados (3, 6 e 12 meses), 40 pacien- tes $>60$ anos com FEPU submetidos ao tratamento não operatório. Foram utilizadas as escalas da ASES, Constant-Murley e Single Assessment Numeric Evaluation (SANE, na sigla em inglês). As variáveis radiográficas incluíram as classificações de Neer e Resch, a presença de fratura e desvio dos tubérculos, cominuição metafisária, lesão periosteal medial, desvios angulares e translacionais da cabeça no plano coronal e sagital e desvio \\ Palavras-chave \\ - fraturas do ombro \\ - complicações \\ - tratamento conservador \\ - diagnóstico por imagem dos tubérculos. \\ Resultados Observamos resultados pela escala de ASES de 77,7 $\pm 23,2$ para toda a amostra, pela de Constant-Murley de $68,7 \pm 16$ e de $82,6 \%$ para a escala em relação ao lado contralateral. A escala de SANE aos 12 meses foi de $84,8 \pm 19$. Os critérios radiográficos que apresentaram influência negativa no resultado clínico pela escala de ASES aos 12 meses foram a gravidade pela classificação de Neer e pelo desvio angular no plano coronal (mensurado pelo ângulo cabeça-diáfise) e a presença de fratura dos tubérculos.}

Trabalho desenvolvido no Instituto de Ortopedia e Traumatologia Hospital das Clínicas, Faculdade de Medicina, Universidade de São Paulo, São Paulo, SP, Brasil. recebido

07 de Março de 2020

aceito

06 de Julho de 2020

Publicado on-line

Novembro 2, 2020
DOI https://doi.org/

10.1055/s-0040-1716760. ISSN $0102-3616$. (c) 2020. Sociedade Brasileira de Ortopedia e Traumatologia. All rights reserved.

This is an open access article published by Thieme under the terms of the Creative Commons Attribution-NonDerivative-NonCommercial-License, permitting copying and reproduction so long as the original work is given appropriate credit. Contents may not be used for commercial purposes, or adapted, remixed, transformed or built upon. (https://creativecommons.org/ licenses/by-nc-nd/4.0/)

Thieme Revinter Publicações Ltda., Rua do Matoso 170, Rio de Janeiro, RJ, CEP 20270-135, Brazil 


\author{
Abstract \\ Keywords \\ - shoulder fractures \\ - complications \\ - conservative \\ treatment \\ - diagnostic imaging
}

Conclusão O tratamento não operatório de fraturas desviadas da extremidade proximal do úmero em pacientes idosos resulta em bons resultados clínicos. Os resultados clínicos são influenciados negativamente pelo desvio angular da cabeça do úmero e pela presença de fratura dos tubérculos maior e menor, assim como pela classificação de Neer.

Objectives To describe the functional result of the conservative treatment of displaced proximal humerus fractures (PHF) using the American Shoulder and Elbow Surgeons (ASES) score after 12 months and assess whether the different initial classifications and radiographic measurements are related to clinical results.

Methods Forty patients $>60$ years old, with displaced PHUs submitted to conservative treatment were evaluated at standardized times (3, 6, and 12 months). The American Society of Shoulder and Elbow Surgeons (ASES), Constant-Murley and Single Assessment Numeric Evaluation (SANE) scales were used as clinical outcomes. Radiographic variables included the Neer and Resch classifications, the presence and displacement of tuberosity fracture, metaphyseal comminution, medial periosteal lesion, and angular and translational deviations of the head in the coronal and sagittal plane.

Results The result of the ASES score was $77.7 \pm 23.2$ for the whole sample, the mean absolute values of the Constant-Murley score were $68.7 \pm 16$ and $82.6 \%$ for the scale relative to the contralateral side. The SANE scale at 12 months was $84.8 \pm 19$. We observed that the severity of the Neer classification and the coronal plane angular deviation (measured by the head-shaft angle) and the presence of fractures in both tuberosities negatively influenced the ASES score after 12 months of treatment.

Conclusion Nonoperative treatment of displaced proximal humerus fractures in elderly patients results in good clinical results. Clinical results are negatively influenced by the angular deviation of the humeral head and the presence of fractures of the greater and lesser tubercles, as well as by the Neer classification.

\section{Introdução}

Fraturas relacionadas à osteoporose ocorrem em $\sim 2$ milhões de indivíduos por ano nos EUA, e as fraturas da extremidade proximal do úmero (FEPUs) são responsáveis por $10 \%$ destas. ${ }^{1}$ Estudos recentes demonstram que o tratamento não operatório leva a resultados semelhantes ao cirúrgico, independentemente da idade e do padrão da fratura. ${ }^{2,3}$

No entanto, ainda existem controvérsias sobre os principais padrões que se beneficiariam do tratamento cirúrgico, $\mathrm{e}$ faltam evidências sobre os parâmetros radiográficos de pior prognóstico para o tratamento não operatório.

As classificações de Neer, Arbeitsgemeinschaft für Osteosynthesefragen (AO) e binária apresentam baixa confiabilidade e pouco acrescentam à tomada de decisão do tratamento. ${ }^{4-6}$ Estudos prévios demonstram que diversas características relacionadas à fratura, e não avaliadas por estas classificações, podem influenciar os resultados funcionais, como a cominuição metafisária medial, ${ }^{7}$ o tipo e grau de desvio no plano coronal e sagital e a perda óssea por impacção. ${ }^{8}$ Outros fatores de prognóstico estão relacionados ao paciente e à lesão, como a idade, osteoporose e o tempo entre a fratura e o tratamento. ${ }^{9,10}$
Poucos estudos avaliam os diferentes critérios radiográficos para prever os resultados funcionais no tratamento não operatório das fraturas desviadas da extremidade proximal do úmero. ${ }^{8,11-15}$ Além disso, nenhum estudo prévio avaliou os critérios de Resch e outras variáveis radiográficas no prognóstico das FEPUs.

O objetivo primário do presente estudo é descrever o resultado funcional do tratamento não operatório de fraturas desviadas da extremidade proximal do úmero pela escala da American Shoulder and Elbow Surgeons (ASES, na sigla em inglês) após 12 meses. 0 objetivo secundário é avaliar se as diferentes classificações e medidas radiográficas iniciais têm relação com piores resultados pela escala ASES.

\section{Métodos}

\section{Desenho do estudo}

Realizamos um estudo prospectivo envolvendo 40 pacientes com FEPUs, submetidos ao tratamento não operatório. Os pacientes fazem parte de um estudo randomizado e foram tratados entre fevereiro de 2016 e outubro de 2018 em um único centro. 0 protocolo foi aprovado pela Comissão de Ética de nosso serviço sob o parecer número 1.266.876. 


\section{Participantes}

Os critérios de inclusão foram idade $\geq 60$ anos, $<30$ dias do trauma e FEPU com envolvimento do colo cirúrgico, com pelo menos um dos seguintes parâmetros: ângulo cabeçadiáfise com desvio $\geq 20^{\circ}$ (varo ou valgo); translação cabeçadiáfise $>1 \mathrm{~cm}$ (no plano frontal ou sagital) e fratura associada do tubérculo maior e/ou menor com desvio $>0,5 \mathrm{~cm}$. Os critérios foram avaliados por dois médicos especialistas em cirurgia do ombro e cotovelo antes da inclusão no estudo.

Não foram incluídas as fraturas-luxação e as fraturas com ausência de contato entre a cabeça e a diáfise e as bilaterais. Não foram incluídos pacientes com fratura associada no membro superior ipsi ou contralateral, com lesões neurológicas diagnosticadas pelo exame físico, fraturas associadas no membro acometido, fraturas patológicas, fraturas bilaterais, cirurgias prévias no ombro acometido ou rotura de espessura completa de um dos tendões do manguito rotador diagnosticada previamente.

\section{Intervenção}

O tratamento não operatório consistiu no uso da tipoia velpeau associado a reabilitação precoce. Nenhum paciente foi submetido a redução incruenta. A analgesia foi padronizada, com o uso de dipirona $500 \mathrm{mg}$ (um comprimido a cada $8 \mathrm{~h}$ por 10 dias), codeína $30 \mathrm{mg}$ (um comprimido a cada $6 \mathrm{~h}$ por 7 dias e depois conforme a dor) e paracetamol $500 \mathrm{mg}$ (um comprimido a cada 8 h por 10 dias).

O uso da tipoia foi orientado de modo padronizado e um panfleto impresso foi entregue aos pacientes após a inclusão no estudo, contendo as orientações de reabilitação descritas abaixo. Os exercícios domiciliares foram orientados pessoalmente por um fisioterapeuta no $1^{\circ}$ dia de inclusão no estudo.

A movimentação do cotovelo, punho e mão foi iniciada no $1^{\circ}$ dia de inclusão no estudo. Foram orientados modos de realização da higiene pessoal, posição para dormir e para a alimentação. Foram orientados exercícios domiciliares cervicais, escapulares, de cotovelo, punho e mão no $1^{\circ}$ dia de inclusão no estudo e os exercícios pendulares no $7^{\circ}$ dia. Em 15 dias, foram iniciados exercícios passivos do ombro para elevação, abdução e para as rotações. Após 30 dias, foram iniciados exercícios ativo-resistidos de cotovelo, ativo-assistidos e ativo-livres do ombro e exercícios isométricos para o manguito, o deltóide e a cintura escapular, conforme tolerado pelos pacientes. Exercícios ativo-resistidos para o manguito, o deltóide e a cintura escapular foram iniciados no $45^{\circ}$ dia. 0 tempo máximo indicado de uso da tipoia foi de 30 dias após a fratura.

\section{Desfechos}

Foi adotado como desfecho primário a avaliação clínica pela escala ASES após 12 meses da fratura. Foram considerados desfechos secundários: a escala de Constant-Murley, em seus valores absolutos e relativos ao membro contralateral (CRI) e a escala Single Assessment Numeric Evaluation (SANE, na sigla em inglês). As escalas foram aplicadas em 3, 6 e 12 meses após a fratura. A aplicação das escalas clínicas foi realizada por um avaliador que não participava da reabilitação ou do seguimento clínico do paciente.

As radiografias foram realizadas em quatro incidências, sempre que tolerado pelo paciente, incluindo o frente verdadeiro, perfil verdadeiro, perfil axilar e incidência de Velpeau. As radiografias foram avaliadas com o sistema de armazenamento de imagens da instituição (iSite enterprise 4.1, Phillips, Amsterdã, Holanda). Exame de tomografia computadorizada foi realizado em todos os pacientes antes da inclusão no estudo. Os resultados foram utilizados para complementar a definição das variáveis radiográficas da fratura, como as classificações e características dos desvios. Dois ortopedistas, membros da Sociedade Brasileira de Ombro e Cotovelo, com 11 e 12 anos de experiência, avaliaram todas as imagens e as classificações foram determinadas por consenso. As medidas utilizadas foram as de um dos avaliadores.

Exame de ultrassonografia foi realizado aos 6 meses de pós-operatório para a avaliação da integridade do manguito rotador. Os exames foram realizados no departamento de radiologia da instituição pela equipe de radiologistas musculoesqueléticos. Foi utilizado o aparelho Logiq E9 (GE Healthcare, Waukesha, WI, EUA), com transdutor linear ML6-15 (6- to 15-MHz linear ML-6-15, GE Healthcare, Waukesha, WI, EUA). Os achados relacionados aos tendões do manguito rotador foram classificados de acordo com a presença de rotura transfixante de um ou mais tendões.

As complicações foram anotadas de acordo com sua ocorrência e o número total registrado separadamente para cada paciente. Não consideramos consolidação viciosa como uma complicação. A necessidade de abordagem cirúrgica e o tipo de cirurgia realizada também foram registradas. As seguintes complicações foram analisadas como variáveis binárias (presente ou ausente), sendo consideradas presentes se identificadas em qualquer período do tratamento: dor recorrente com limitação funcional com necessidade de tratamento adicional (clínico ou cirúrgico) após 1 ano de tratamento; intercorrência clínica relacionada à fratura ou tratamento com necessidade de internação; óbito relacionado à fratura ou tratamento; refratura: rigidez do ombro, definida pela diminuição da amplitude de movimento do ombro com limitação funcional mantida após 6 meses da fratura; síndrome complexa de dor regional; ossificação heterotópica; osteoartrose glenoumeral; osteonecrose da cabeça do úmero e pseudoartrose, definida pela ausência de consolidação após 1 ano da fratura. Exames complementares (tomografia computadorizada, ressonância magnética [RM] ou eletroneuromiografia) foram realizados de acordo com a suspeita clínica.

\section{Variáveis analisadas}

As variáveis clínicas avaliadas foram: gênero, lado, dominância, tabagismo, diabetes, tempo até o início da reabilitação, duração da reabilitação e número de sessões.

As variáveis radiográficas incluíram as classificações de Neer e Resch, a presença de fratura dos tubérculos (independente do desvio), cominuição metafisária e lesão periosteal medial. Foram avaliados diversos parâmetros radiográficos de desvio da fratura. 0 ângulo cabeça-diáfise foi avaliado conforme previamente publicado. ${ }^{16} \mathrm{O}$ desvio do tubérculo maior 
foi avaliado de acordo com a sua distância em relação ao ápice da cabeça do úmero, na radiografia em frente verdadeiro. 0 tubérculo menor foi avaliado na radiografia axilar ou velpeau quanto ao desvio do fragmento. As demais medidas foram avaliadas em relação ao desvio dos fragmentos. Todas as medidas foram categorizadas em 3 níveis, de acordo com critérios previamente estabelecidos. A - Tabela 1 descreve todas as variáveis radiográficas analisadas.

\section{Análise estatística}

Devido ao tamanho da amostra, optou-se por testes não paramétricos, independentemente da normalidade dos dados. Apresentamos as variáveis contínuas em médias e desvio padrão(DP)

Tabela 1 Descrição dos critérios de desvio radiográfico analisados

\begin{tabular}{|c|c|}
\hline \multicolumn{2}{|c|}{ Cominuição metafisária } \\
\hline Não & \\
\hline Sim & \\
\hline Lesão periosteal mec & \\
\hline Não & \\
\hline Sim & \\
\hline Fratura dos tubércul & \\
\hline Sem fratura dos tu & érculos \\
\hline Tubérculo maior & \\
\hline Tubérculos maior - & menor \\
\hline Desvio do tubérculo & haior \\
\hline Até $2 \mathrm{~mm}$ inferior & o topo da cabeça do úmero \\
\hline Até $2 \mathrm{~mm}$ superior & ao topo de cabeça \\
\hline Acima de $2 \mathrm{~mm} d c$ & topo de cabeça \\
\hline Desvio do tubérculo & nenor \\
\hline Até 2 mm & \\
\hline Entre 2 e $5 \mathrm{~mm}$ & \\
\hline$>5 \mathrm{~mm}$ & \\
\hline Ângulo cabeça diáfis & frente \\
\hline $125^{\circ}$ a $150^{\circ}$ & \\
\hline $110^{\circ}$ a $124^{\circ}$ ou 15 & a $165^{\circ}$ \\
\hline$<110^{\circ}$ ou $>166^{\circ}$ & \\
\hline Ângulo cabeça diáfis & perfil \\
\hline Sem desvio & \\
\hline Moderado $\left(0-20^{\circ}\right)$ & \\
\hline Grave $\left(>45^{\circ}\right)$ & \\
\hline Desvio diáfise frente & \\
\hline Até $5 \mathrm{~mm}$ & \\
\hline Entre 5 e $10 \mathrm{~mm}$ & \\
\hline$>10 \mathrm{~mm}$ & \\
\hline Desvio diáfise perfil & \\
\hline Até $5 \mathrm{~mm}$ & \\
\hline Entre 5 e $10 \mathrm{~mm}$ & \\
\hline$>10 \mathrm{~mm}$ & \\
\hline
\end{tabular}

e medianas e intervalo interquartil. As categóricas foram apresentadas em valor absoluto e percentual. O cálculo de amostra foi por conveniência, com casos incluídos sequencialmente.

A comparação das escalas funcionais antes e após o tratamento foi realizada pelo teste de Wilcoxon. Para as análises univariadas com as variáveis radiográficas, utilizamos o teste Kruskal-Wallis e o teste de Friedman para a análise post-hoc.

Foi utilizado para análise dos dados o programa IBM SPSS Statistics for Windows, versão 20.0 (IBM Corp., Armonk, NY, USA) e adotado o nível de significância de $5 \%$.

\section{Resultados}

Foram incluídos 45 pacientes para o tratamento não operatório. Cinco pacientes não realizaram a primeira avaliação aos 3 meses, com perda completa do seguimento. Foram incluídos 40 pacientes com 12 meses de seguimento completos na avaliação final. Os dados gerais da amostra podem ser vistos na - Tabela 2, sendo que a maioria da amostra consistiu em mulheres, com média de idade de $69 \pm 6$ anos. As - Figuras 1, 2 e 3 demonstram o resultado radiográfico e clínico de um paciente. O tempo médio até o início da fisioterapia foi de $21 \pm 12$ dias, com uma média de $111 \pm 51$ dias de duração da reabilitação e $18 \pm 10$ sessões. Os pacientes que iniciaram a fisioterapia com $>15$ dias da fratura apresentaram média de $72 \pm 25$ pontos pela escala ASES comparados à média de $79,9 \pm 23,4$ pontos para os pacientes que iniciaram com $\leq 15$ dias.

Os resultados pela escala ASES aos 12 meses foram de $77,7 \pm 23,2$ pontos para toda a amostra, com melhora ao

Tabela 2 Características clínicas dos pacientes submetidos ao tratamento não operatório de fraturas desviadas da extremidade proximal do úmero

\begin{tabular}{|l|l|l|}
\hline & $n$ & $\%$ \\
\hline Gênero & & \\
\hline Masculino & 7 & 17.5 \\
\hline Feminino & 33 & 82.5 \\
\hline Lado & & \\
\hline Direito & 13 & 32.5 \\
\hline Esquerdo & 27 & 67.5 \\
\hline Lado dominante acometido & & \\
\hline Sim & 15 & 37.5 \\
\hline Não & 25 & 62.5 \\
\hline Tabagismo & & \\
\hline Não & 31 & 77.5 \\
\hline Tabagista & 7 & 17.5 \\
\hline Ex-tabagista & 2 & 5.0 \\
\hline Diabetes & & \\
\hline Não & 30 & 75.0 \\
\hline Sim, não insulino dependente & 8 & 20.0 \\
\hline Sim, insulino dependente & 2 & 5.0 \\
\hline
\end{tabular}



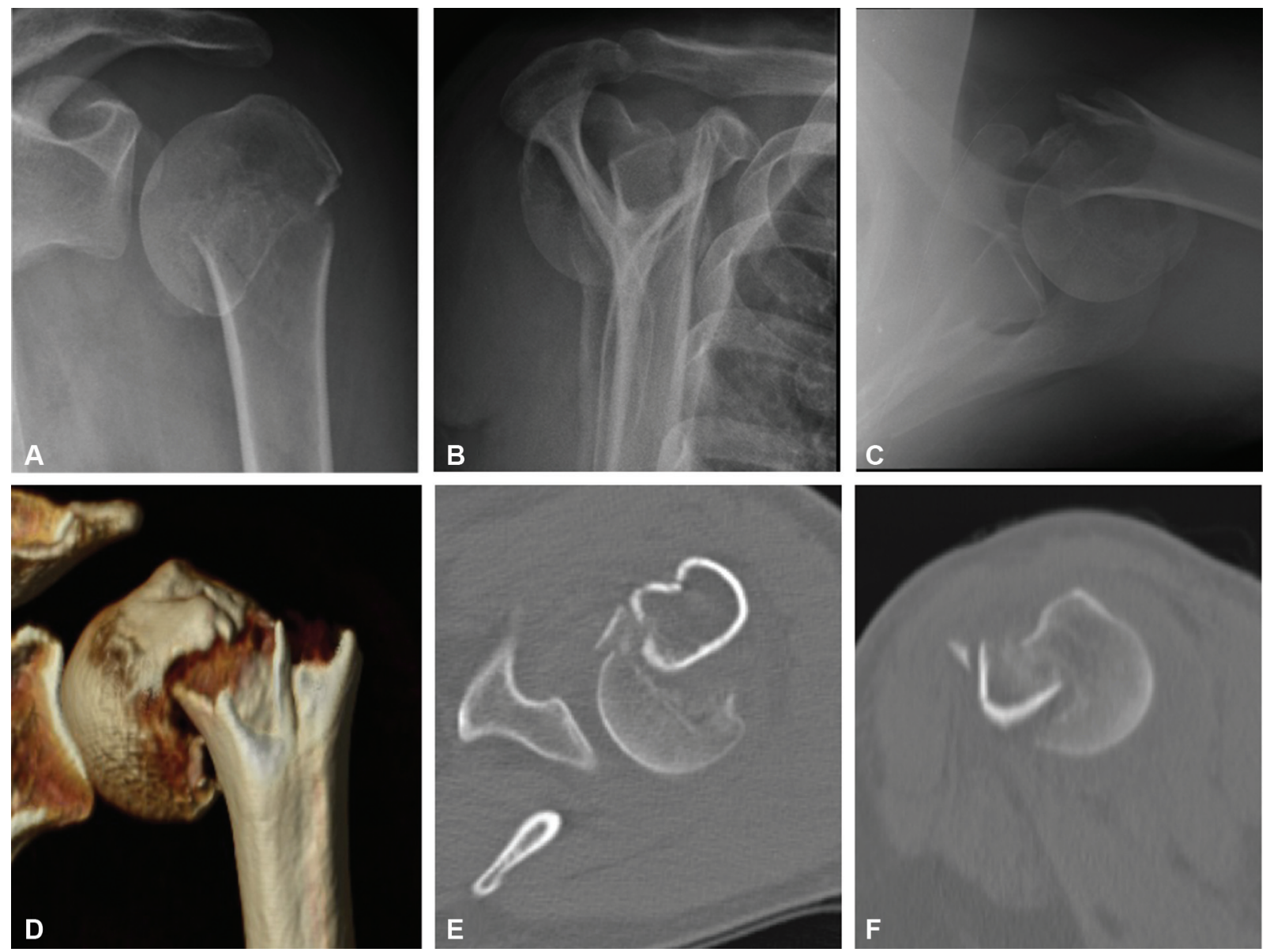

Fig. 1 Radiografias iniciais na incidência em frente verdadeiro (A), perfil (B) e axilar (C). Tomografia computadorizada demonstrando a fratura com reconstrução tridimensional (D), corte axial (E) e sagital (F).

longo do tempo. Aos 12 meses, a média dos valores absolutos da escala de Constant-Murley foi de $68,7 \pm 16$ pontos e de $82,6 \%$ para a escala em relação ao lado contralateral. A escala de SANE aos 12 meses foi de $84,8 \pm 19$ pontos. Os resultados clínicos podem ser vistos na - Tabela 3 .

A ultrassonografia foi realizada em 37 pacientes (92,5\%). Roturas transfixantes do manguito rotador foram observadas em 8 pacientes $(21,6 \%)$. Os pacientes com roturas do manguito apresentaram média de $76,9 \pm 24,0$ pontos pela escala ASES aos 12 meses, sem diferença estatisticamente significativa para os pacientes sem lesões do manguito $(p=0,188)$.

Complicações ocorreram em 8 pacientes (20\%), que incluíram 4 casos de osteonecrose (10\%), 2 casos de pseudoartrose (5\%) e 2 casos de rigidez persistente (5\%). Nenhum paciente desejou abordagem cirúrgica para o tratamento das complicações. A - Figura 3 demonstra um caso com osteonecrose e a -Figura 4 um caso de pseudoartrose. Os pacientes com complicações apresentaram média de 53,8 $\pm 23,0$ pontos pela escala ASES.

A análise das variáveis radiográficas demonstrou a influência negativa dos seguintes parâmetros no resultado clínico pela escala de ASES aos 12 meses: gravidade pela classificação de Neer e pelo desvio angular no plano coronal (mensurado pelo ângulo cabeça-diáfise) e a presença de fratura dos tubérculos. As demais variáveis radiográficas não influenciaram no resultado funcional pela escala ASES. Os resultados da análise de subgrupo para as principais variáveis são demonstrados na - Tabela 4.

\section{Discussão}

Nossos resultados demonstram que o tratamento não operatório pode ser realizado mesmo em fraturas com desvio significativo dos tubérculos ou da cabeça. Obtivemos resultados da escala de Constant-Murley relativa ao lado contralateral de $82,6 \%$, valores semelhantes aos de outros estudos sobre tratamento não operatório, ${ }^{8,11-14}$ assim como os obtidos no tratamento cirúrgico com artroplastia ou com a fixação com placa bloqueada. ${ }^{17-20}$ Nas escalas subjetivas, ASES e SANE, obtivemos valores semelhantes ao demonstrado nas revisões sistemáticas. $^{2}$ Nossos resultados reforçam os achados do estudo Proximal Fracture of the Humerus Evaluation by Randomization (PROFHER) e da revisão sistemática Cochrane, demonstrando que o tratamento não operatório, mesmo em fraturas desviadas, pode trazer bons resultados funcionais., ${ }^{2,3}$

Em revisão sistemática, Sabharwal et al. ${ }^{21}$ demonstram resultados clínicos semelhantes para ambos os tratamentos. No entanto, demonstram que para as fraturas mais complexas, como as em 4 partes, o tratamento cirúrgico apresenta melhores resultados clínicos e menor taxa de complicações. Os autores 

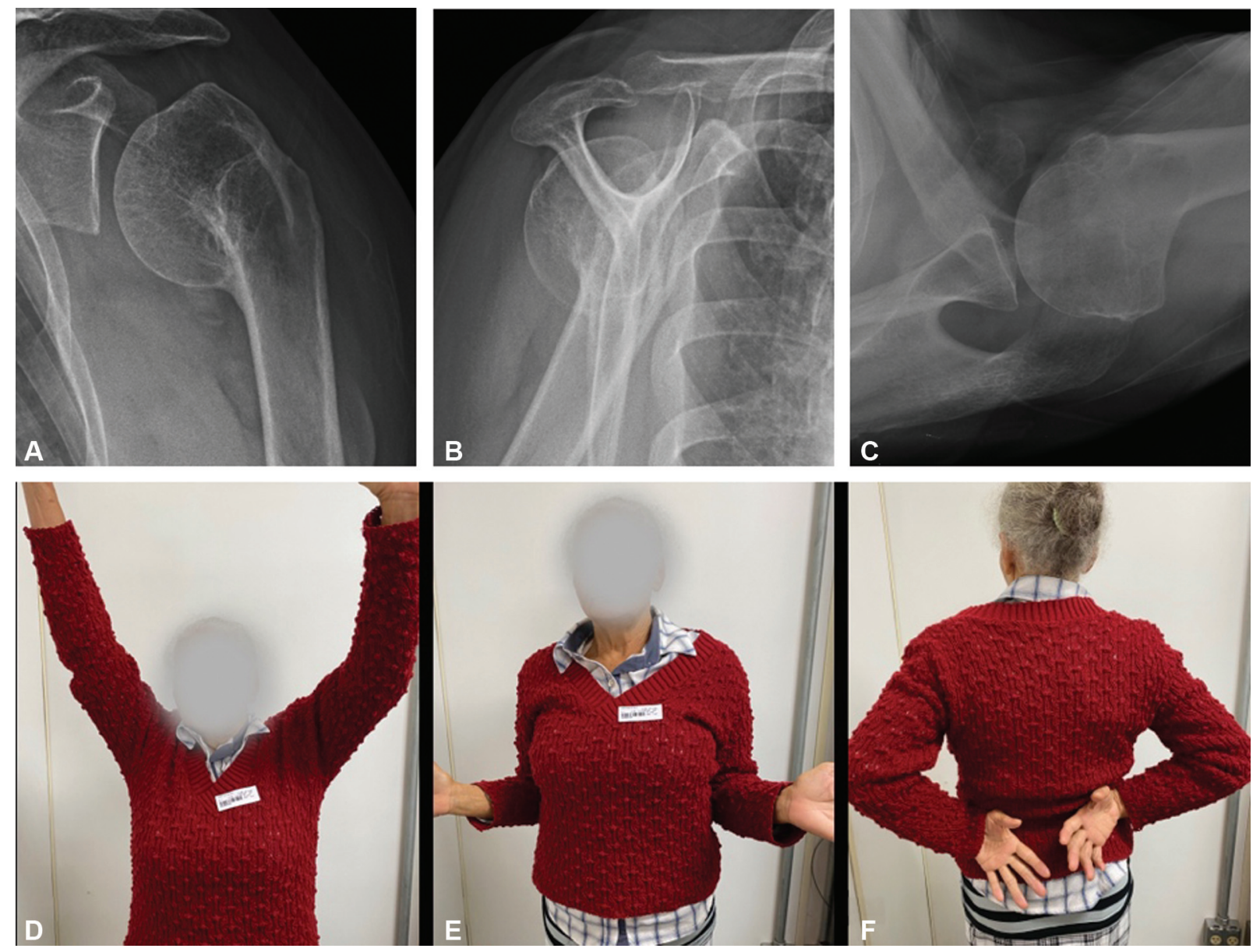

Fig. 2 Resultado após 1 ano, demonstrando a consolidação da fratura e o desvio em varo na incidência em frente verdadeiro (A), perfil (B) e axilar (C). Resultado clínico demonstrando a elevação ativa (D), rotação lateral ativa (E) e rotação medial ativa (F).
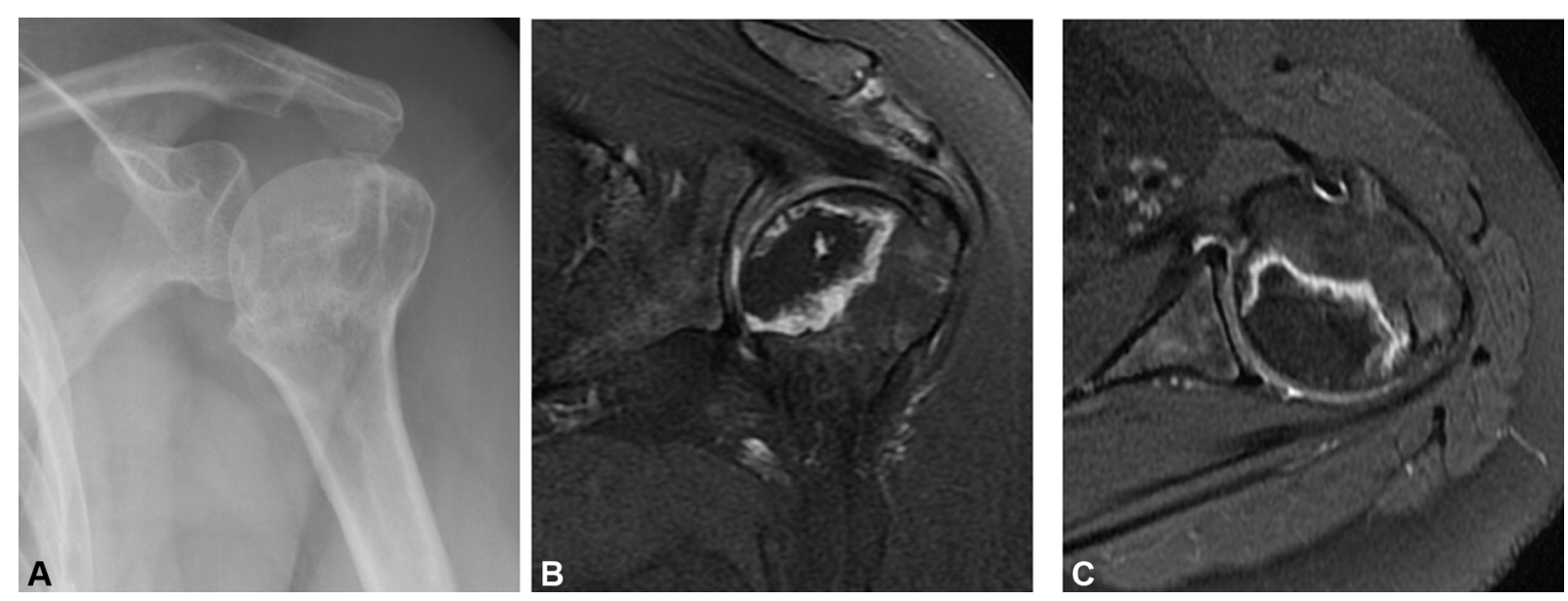

Fig. 3 Radiografia (a) e ressonância magnética (b) demonstrando um caso de osteonecrose da cabeça do úmero.

destacam a necessidade de futuros estudos com subtipos específicos de fraturas.

Poucos estudos avaliaram a influência da classificação e dos critérios de desvio definidos por Neer no tratamento não operatório das FEPU. ${ }^{8,14}$ Apesar de serem rotineiramente utilizados como critérios para a indicação do tratamento cirúrgico, os valores de desvio angular de $45^{\circ}$, de desvio translacional de $1 \mathrm{~cm}$ e de desvio do tubérculo de $0,5 \mathrm{~cm}$ foram arbitrariamente definidos, conforme explicado em publicação do próprio Neer em 2002.22

O objetivo secundário do nosso estudo foi avaliar não só os critérios de desvio descritos por Neer, assim como diversos outros parâmetros radiográficos. A classificação recentemente descrita por Resch et al. ${ }^{23}$ apresenta melhor confiabilidade inter- e intra-observador quando comparada à 
Tabela 3 Resultados das escalas funcionais aos 3, 6 e 12 meses de seguimento após o tratamento não operatório de fraturas desviadas da extremidade proximal do úmero

\begin{tabular}{|c|l|l|l|l|l|}
\hline & $\boldsymbol{n}$ & Média & DP & Mediana & IIQ \\
\hline ASES & & & & & \\
\hline 3 meses & 39 & 57,7 & 24,5 & 56,3 & 42,8 \\
\hline 6 meses & 39 & 71,6 & 24,3 & 80,7 & 36,0 \\
\hline 12 meses & 40 & 77,7 & 23,2 & 85,2 & 40,1 \\
\hline SANE & & & & & \\
\hline 3 meses & 39 & 66,2 & 22,6 & 70,0 & 40,0 \\
\hline 6 meses & 39 & 82,2 & 19,4 & 85,0 & 22,5 \\
\hline 12 meses & 40 & 84,8 & 19,0 & 90,0 & 20,0 \\
\hline Constant-Murley & 39 & 55,6 & 16,7 & 60,0 & 31,0 \\
\hline 3 meses & 39 & 15,9 & 68,5 & 28,5 \\
\hline 6 meses & 40 & 64,8 & 16,0 & 72,0 & 24,0 \\
\hline 12 meses & 40 & 68,7 & & \\
\hline Constant relativo contralateral \\
\hline 6 meses & 39 & $80,1 \%$ & $18,7 \%$ & $87,2 \%$ & $37,8 \%$ \\
\hline 12 meses & 39 & $82,6 \%$ & $23,6 \%$ & $89,5 \%$ & $31,3 \%$ \\
\hline
\end{tabular}

Abreviações: ASES, Escala da American Shoulder and Elbow Surgeons; DP, Desvio Padrão; IIQ, Intervalo Interquartil; SANE, Single Assessment Numeric Evaluation.

classificação de Neer, mas não foi previamente avaliada quanto ao seu potencial em determinar prognóstico. ${ }^{6}$

$\mathrm{Na}$ análise univariada, pudemos observar diferença estatisticamente significativa e clinicamente relevante para algumas variáveis radiográficas, principalmente quanto ao desvio angular, avaliado pelo ângulo cabeça-diáfise, e quanto pela presença de fratura do tubérculo maior e menor, independente do seu desvio. Pacientes com fraturas dos tubérculos maior e menor apresentaram uma média de 23 pontos a menos na escala ASES, achado de relevância clínica importante. Pudemos observar piores resultados para os pacientes classificados como fraturas desviadas em 4 partes de Neer, semelhante aos achados de Yüksel et al., ${ }^{14}$ porém não observamos diferença estatisticamente significativa entre os classificados como 2 e 3 partes.

A avaliação do ângulo cabeça-diáfise pode ser difícil de ser realizada em radiografias em fraturas agudas e apresenta variável correlação interobservador. ${ }^{16,24}$ Apesar disso, com uma padronização radiográfica adequada, e o eventual uso de tomografia computadorizada (TC), o ângulo cabeça-diáfise é um parâmetro importante para o auxílio da tomada de decisão, pois influencia diretamente os resultados clínicos. Pacientes com angulações $<110^{\circ}$ ou $>166^{\circ}$ apresentaram 29 pontos a menos pela escala ASES quando comparados aos pacientes com mínimo desvio angular $\left(125^{\circ}\right.$ a $\left.150^{\circ}\right)$. Yüksel et al. ${ }^{14}$ não puderam observar essa influência com a escala de Constant-Murley, com resultados semelhantes entre as fraturas impactadas em valgo ou as em varo $<110^{\circ}$.

A comparação apenas das médias desse ângulo pode levar a interpretações incorretas quando são incluídas as fraturas com desvio em varo e em valgo, pois aproxima valores em extremos opostos. A análise categorizada, como a realizada em nosso estudo, ou separada pelos desvios em valgo ou varo, deve ser realizada para evitar vieses em sua avaliação. Em contraste aos achados de Court-Brown et al., ${ }^{12}$ em nossa amostra os pacientes com fraturas desviadas em valgo grave $\left(>166^{\circ}\right)$ apresentaram piores médias da escala ASES que os pacientes com varo grave $\left(<110^{\circ}\right)$, porém sem diferença estatisticamente significante. Com achados semelhantes aos nossos, Foruria et al. ${ }^{8}$ demonstraram que as fraturas impactadas em valgo apresentaram risco 3 vezes maior de piora de 10 pontos na escala Disabilities of the Arm, Shoulder and Hand (DASH, na sigla em inglês), quando comparadas às impactadas em varo.

Outros fatores não apresentaram diferença estatisticamente significante, sendo os de maior destaque a presença ou não de cominuição metafisária, a lesão periosteal medial e os desvios no plano sagital. Alguns estudos ${ }^{25,26}$ observaram piores resultados quanto à estabilidade após fixação com placa para os pacientes com cominuição metafisária
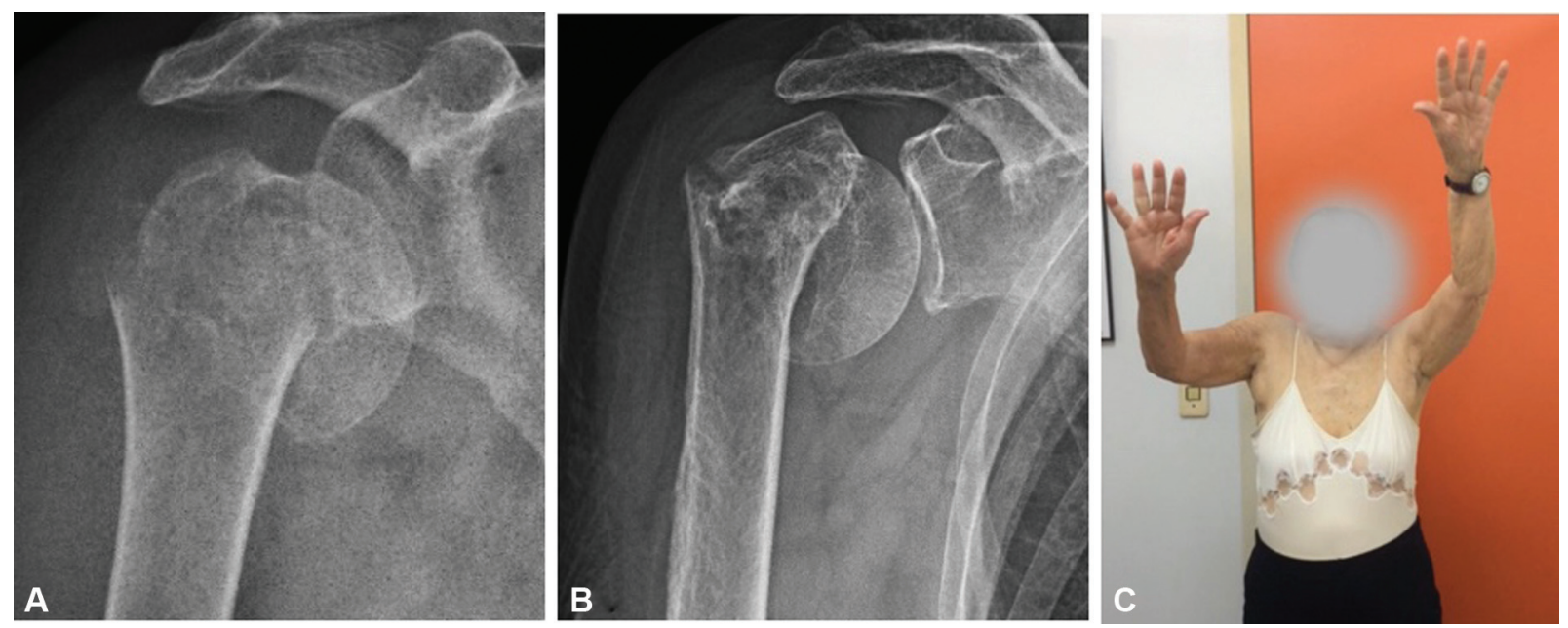

Fig. 4 Radiografia inicial (A) e aos 12 meses (B) de uma paciente com pseudoartrose do colo do úmero. Exame clínico (C) demonstrando elevação ativa máxima. 
Tabela 4 Resultados da análise de subgrupo para as principais variáveis radiográficas e classificações

\begin{tabular}{|c|c|c|c|c|c|}
\hline & \multicolumn{4}{|c|}{ ASES 12 meses } & \multirow[b]{2}{*}{ valor-p } \\
\hline & Média & DP & Mediana & IIQ & \\
\hline \multicolumn{6}{|l|}{ Classificação de Neer } \\
\hline 2 partes & 88,4 & 15,4 & 94,2 & 19,9 & \\
\hline 3 partes & 79,0 & 21,5 & 85,2 & 32,3 & \\
\hline 4 partes & 61,0 & 28,3 & 47,5 & 56,0 & 0,031 \\
\hline \multicolumn{6}{|c|}{ Ângulo cabeça diáfise - coronal } \\
\hline $125^{\circ}$ a $150^{\circ}$ & 93,4 & 7,8 & 96,3 & 11,9 & \\
\hline $\begin{array}{l}110^{\circ} \text { a } 124^{\circ} \text { ou } \\
151^{\circ} \text { a } 165^{\circ}\end{array}$ & 74,5 & 25,2 & 78,4 & 45,4 & \\
\hline$<110^{\circ}$ ou $>166^{\circ}$ & 64,3 & 25,0 & 56,5 & 48,2 & 0,004 \\
\hline \multicolumn{6}{|l|}{$\begin{array}{l}\text { Desvio angular - } \\
\text { coronal }\end{array}$} \\
\hline Pouco desvio & 84,7 & 20,4 & 93,8 & 26,1 & \\
\hline $\begin{array}{l}\text { Valgo grave } \\
\left(>166^{\circ}\right)\end{array}$ & 60,0 & 22,3 & 58,1 & 41,9 & \\
\hline $\begin{array}{l}\text { Varo grave } \\
\left(<110^{\circ}\right)\end{array}$ & 66,2 & 27,1 & 56,5 & 54,9 & 0,034 \\
\hline \multicolumn{6}{|c|}{ Fratura dos tubérculos } \\
\hline $\begin{array}{l}\text { Sem fratura dos } \\
\text { tubérculos }\end{array}$ & 84,7 & 19,7 & 94,2 & 38,4 & \\
\hline Tubérculo maior & 82,8 & 20,3 & 88,1 & 27,5 & \\
\hline $\begin{array}{l}\text { Tubérculos } \\
\text { maior + menor }\end{array}$ & 61,5 & 26,9 & 50,4 & 54,6 & 0,031 \\
\hline \multicolumn{6}{|c|}{ Classificação de Resch } \\
\hline 1 & 94,2 & 6,4 & 95,9 & 12,6 & \\
\hline 2 & 88,1 & 16,8 & 88,1 & $\mathrm{NA}$ & \\
\hline 3 & 64,4 & 27,1 & 61,5 & 53,0 & \\
\hline 4 & 76,2 & 23,7 & 84,9 & 44,8 & 0,052 \\
\hline \multicolumn{6}{|l|}{$\begin{array}{l}\text { Cominuição } \\
\text { metafisária }\end{array}$} \\
\hline Não & 75,8 & 23,7 & 85,4 & 49,7 & \\
\hline Sim & 77,7 & 25,2 & 85,0 & 36,7 & 0,570 \\
\hline \multicolumn{6}{|l|}{$\begin{array}{l}\text { Lesão periosteal } \\
\text { medial }\end{array}$} \\
\hline Não & 82,9 & 22,3 & 93,2 & 27,3 & \\
\hline Sim & 71,6 & 24,7 & 78,5 & 51,7 & 0,168 \\
\hline
\end{tabular}

Abreviações: DP, Desvio Padrão; IIQ, Intervalo Interquartil.

posteromedial, porém nenhum estudo avaliou a importância desse achado no tratamento não operatório.

Não pudemos detectar diferença estatisticamente significante nas escalas funcionais para a classificação descrita por Resch et al. ${ }^{23}$ Apesar disso, as médias dos subtipos em valgo (tipo 3) e varo (tipo 4) foram inferiores aos tipos 1 e 2 .

Variáveis relacionadas à reabilitação não apresentaram diferença em análises de subgrupo, tanto quanto ao tempo até o início da reabilitação, quanto a sua total duração e número de sessões.

Quanto aos achados das lesões do manguito rotador, não observamos relação entre a presença de lesão e piores resultados clínicos. Apesar de não termos incluído pacientes com diagnóstico prévio de lesão do manguito rotador, é possível que alguns dos casos apresentassem lesões assintomáticas, explicando a alta taxa encontrada em nossa amostra. Além disso, o exame de ultrassonografia em pacientes com sequelas de fraturas pode apresentar acurácia inferior, decorrente de limitações de mobilidade ou deformidades.

A presença de roturas do manguito rotador não influenciou o resultado clínico pela escala ASES em nossa amostra.

Nosso estudo apresenta algumas limitações. Nossa amostra é relativamente pequena para análises multivariadas para os diversos fatores radiográficos, sendo optado pela análise univariada, que pode aumentar o risco de fatores confundidores. No entanto, é uma amostra homogênea e constituída por pacientes com características semelhantes.

Como pontos positivos, nosso estudo realizou avaliação clínica e radiográfica prospectiva, em tempos padronizados, com um avaliador não participante do estudo e do acompanhamento dos pacientes. Todas as fraturas incluídas apresentavam desvios significativos, que poderiam ser consideradas de indicação para o tratamento cirúrgico, não sendo incluídas as fraturas sem desvio ou com desvio mínimo nessa avaliação. Nossos resultados podem auxiliar na tomada de decisão entre o tratamento não operatório e o cirúrgico para alguns padrões específicos de fraturas da extremidade proximal do úmero.

\section{Conclusão}

O tratamento não operatório de fraturas desviadas da extremidade proximal do úmero em pacientes idosos resulta em bons resultados clínicos. Os resultados clínicos são influenciados negativamente pelo desvio angular da cabeça do úmero e pela presença de fratura dos tubérculos maior e menor, assim como pela classificação de Neer.

\section{Suporte Financeiro}

Não houve suporte financeiro de fontes públicas, comerciais, ou sem fins lucrativos.

\section{Conflito de Interesses}

Os autores declaram não haver conflito de interesses. Dr. Gracitelli relata honorários pessoais da Arthrex, fora do trabalho submetido.

\section{Referências}

1 Palvanen M, Kannus P, Niemi S, Parkkari J. Update in the epidemiology of proximal humeral fractures. Clin Orthop Relat Res 2006;442(442):87-92

2 Handoll HHG, Brorson S. Interventions for treating proximal humeral fractures in adults. Cochrane Database Syst Rev 2015; (11):CD000434

3 Rangan A, Handoll H, Brealey S, et al. PROFHER Trial Collaborators. Surgical vs nonsurgical treatment of adults with displaced fractures of the proximal humerus: the PROFHER randomized clinical trial. JAMA 2015;313(10):1037-1047

4 Majed A, Macleod I, Bull AMJ, et al. Proximal humeral fracture classification systems revisited. J Shoulder Elbow Surg 2011;20 (07):1125-1132

5 Bernstein J, Adler LM, Blank JE, Dalsey RM, Williams GR, Iannotti JP. Evaluation of the Neer system of classification of proximal 
humeral fractures with computerized tomographic scans and plain radiographs. J Bone Joint Surg Am 1996;78(09):1371-1375

6 Gracitelli MEC, Dotta TAG, Assunção JH, et al. Intraobserver and interobserver agreement in the classification and treatment of proximal humeral fractures. J Shoulder Elbow Surg 2017;26(06): 1097-1102

7 Krappinger D, Bizzotto N, Riedmann S, Kammerlander C, Hengg C, Kralinger FS. Predicting failure after surgical fixation of proximal humerus fractures. Injury 2011;42(11):1283-1288

8 Foruria AM, de Gracia MM, Larson DR, Munuera L, Sanchez-Sotelo $\mathrm{J}$. The pattern of the fracture and displacement of the fragments predict the outcome in proximal humeral fractures. J Bone Joint Surg Br 2011;93(03):378-386

9 Südkamp NP, Audigé L, Lambert S, Hertel R, Konrad G. Path analysis of factors for functional outcome at one year in 463 proximal humeral fractures. J Shoulder Elbow Surg 2011;20(08): 1207-1216

10 Siebenbürger G, Van Delden D, Helfen T, Haasters F, Böcker W, Ockert B. Timing of surgery for open reduction and internal fixation of displaced proximal humeral fractures. Injury 2015; 46(Suppl 4):S58-S62

11 Court-Brown CM, Garg A, McQueen MM. The translated two-part fracture of the proximal humerus. Epidemiology and outcome in the older patient. J Bone Joint Surg Br 2001;83(06):799-804

12 Court-Brown CM, McQueen MM. The impacted varus (A2.2) proximal humeral fracture: prediction of outcome and results of nonoperative treatment in 99 patients. Acta Orthop Scand 2004;75(06):736-740

13 Court-Brown CM, Cattermole H, McQueen MM. Impacted valgus fractures (B1.1) of the proximal humerus. The results of nonoperative treatment. J Bone Joint Surg Br 2002;84(04):504-508

14 Yüksel HY, Yılmaz S, Akşahin E, Celebi L, Muratli HH, Biçimoğlu A. The results of nonoperative treatment for three- and four-part fractures of the proximal humerus in low-demand patients. J Orthop Trauma 2011;25(10):588-595

15 Hanson B, Neidenbach P, de Boer P, Stengel D. Functional outcomes after nonoperative management of fractures of the proximal humerus. J Shoulder Elbow Surg 2009;18(04):612-621
16 Malavolta EA, Assunção JH, Pagotto RA, et al. The rotation of the humeral head does not alter radiographic evaluation of the headshaft angle. J Shoulder Elbow Surg 2016;25(04):543-547

17 Miyazaki AN, Fregoneze M, Santos PD, et al. Results of open reduction and internal fixation of severe fractures of the proximal humerus in elderly patients. Rev Bras Ortop 2014;49(01):25-30

18 Gracitelli MEC, Lobo FL, Ferreira GMA, et al. Outcomes evaluation of locking plate osteosynthesis in displaced fractures of the proximal humerus. Rev Bras Ortop 2013;48(06):491-499

19 Ferrel JR, Trinh TQ, Fischer RA. Reverse total shoulder arthroplasty versus hemiarthroplasty for proximal humeral fractures: a systematic review. J Orthop Trauma 2015;29(01):60-68

20 Brandão BL, Amaral MVG, Cohen M, et al. Treatment of complex acute proximal humerus fractures using hemiarthroplasty. Rev Bras Ortop 2013;48(01):29-35

21 Sabharwal S, Patel NK, Griffiths D, Athanasiou T, Gupte CM, Reilly P. Trials based on specific fracture configuration and surgical procedures likely to be more relevant for decision making in the management of fractures of the proximal humerus: Findings of a meta-analysis. Bone Joint Res 2016;5(10):470-480

22 Neer CS II. Four-segment classification of proximal humeral fractures: purpose and reliable use. J Shoulder Elbow Surg 2002;11(04):389-400

23 Resch H, Tauber M, Neviaser RJ, et al. Classification of proximal humeral fractures based on a pathomorphologic analysis. J Shoulder Elbow Surg 2016;25(03):455-462

24 Assunção JH, Malavolta EA, Beraldo RA, Gracitelli MEC, BordaloRodrigues M, Ferreira Neto AA. Impact of shoulder rotation on neck-shaft angle: A clinical study. Orthop Traumatol Surg Res 2017;103(06):865-868

25 Osterhoff G, Hoch A, Wanner GA, Simmen HP, Werner CML. Calcar comminution as prognostic factor of clinical outcome after locking plate fixation of proximal humeral fractures. Injury 2012;43 (10):1651-1656

26 Ponce BA, Thompson KJ, Raghava P, et al. The role of medial comminution and calcar restoration in varus collapse of proximal humeral fractures treated with locking plates. J Bone Joint Surg Am 2013;95(16):e113, 1-7 